\title{
THE ROLE OF INTERLEUKIN-1B AND INTERLEUKIN-33 IN ATOPIC DERMATITIS
}

\author{
by Rania M. Abdel Hay, Noha F. Ibrahim, Dina Metwally, Laila A. Rashed
}

comment:

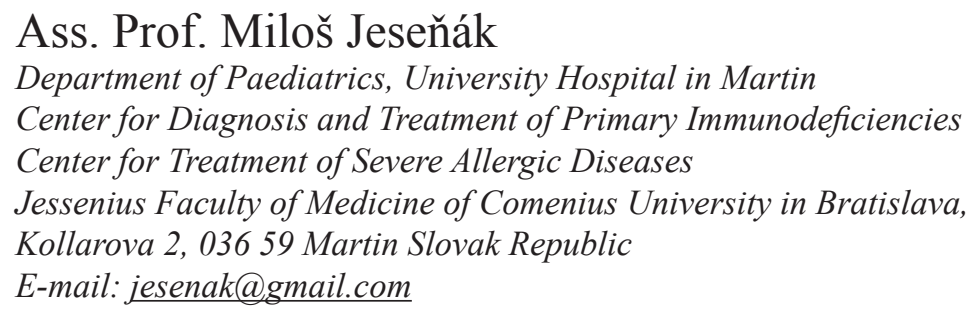

Source of Support: Nil

Competing Interests: None

Our Dermatol Online. 2013; 4(1): 15

Date of submission: 21.11.2012 / acceptance: 30.11 .2012

Cite this article:

Miloš Jeseñák: coment: The role of interleukin-1ß and interleukin-33 in atopic dermatitis. Our Dermatol Online. 2013; 4(1): 15

The studies of the pathogenesis of atopic dermatitis are very important not only for investigation of different aspects of chronic allergic inflammation but also for the possible treatment targets. Authors presented interested in vivo study aimed on the detection of expression of two cytokines (IL-1 $\beta$, IL-33) in the skin of the subjects with moderate to severe atopic dermatitis [1]. Interleukin 33 is one of the newly revealed inflammatory TH2 cytokines which is now studied in relationship with the development of different allergic conditions. The study is of great value, because it examined the production of these two cytokines in vivo in the skin of the dermatitis patients. The role of IL-1 $\beta$ in the chronic inflammation was previously reported, but the finding of increased IL-33 is new. Its association with the severe forms of atopic dermatitis could suggest on one side its role in the progression and exacerbation of the disease, and on the other hand it could be used as possible targeted of aimed biological therapy in the future.

\section{REFERENCES}

1. Abdel Hay RM, Ibrahim NF, Metwally D, Rashed LA: The role of interleukin- $1 \beta$ and interleukin-33 in atopic dermatitis. Our Dermatol Online. 2013;4:11-14. 